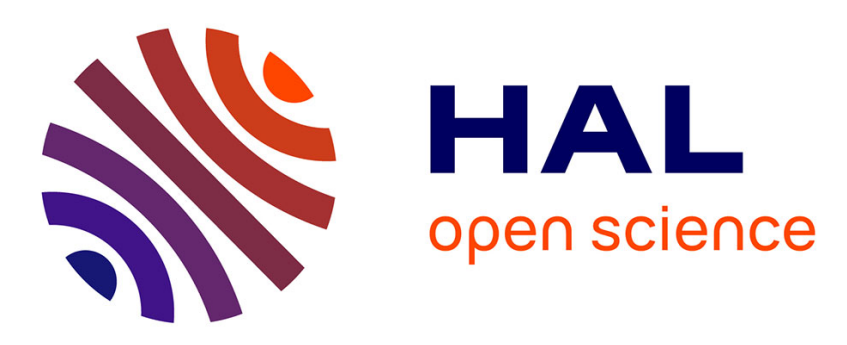

\title{
Démonstration du ciblage des sinus maxillaires par aérosolthérapie sonique
}

\author{
Marc Durand, Sandrine Le Guellec, Jérémie Pourchez, Francis Dubois, Gérald
}

Aubert, Gilles Chantrel, Laurent Vecellio, Cloé Hupin, Ruth de Gersem, Grégory Reychler, et al.

\section{To cite this version:}

Marc Durand, Sandrine Le Guellec, Jérémie Pourchez, Francis Dubois, Gérald Aubert, et al.. Démonstration du ciblage des sinus maxillaires par aérosolthérapie sonique. Annales françaises d'Oto-rhinolaryngologie et de Pathologie Cervico-faciale, 2012, 129 (5), pp.288-295. 10.1016/j.aforl.2011.12.002 . hal-00757521

\section{HAL Id: hal-00757521 \\ https://hal.science/hal-00757521}

Submitted on 28 Nov 2012

HAL is a multi-disciplinary open access archive for the deposit and dissemination of scientific research documents, whether they are published or not. The documents may come from teaching and research institutions in France or abroad, or from public or private research centers.
L'archive ouverte pluridisciplinaire HAL, est destinée au dépôt et à la diffusion de documents scientifiques de niveau recherche, publiés ou non, émanant des établissements d'enseignement et de recherche français ou étrangers, des laboratoires publics ou privés. 
Démonstration du ciblage des sinus maxillaires par aérosolthérapie sonique

Sonic aerosoltherapy to target maxillary sinuses

Marc Durand $(1,2,3,4)^{*}$, Sandrine Le Guellec $(5,6)$, Jérémie Pourchez $(3,7)$, Francis Dubois (2,3,8), Gérald Aubert (9), Gilles Chantrel (10), Laurent Vecellio (5,6), Chloé Hupin (11), Ruth De Gersem (12), Gregory Reychler (13), Laurent Pitance (13), Patrice Diot (6), François Jamar (12)

1-Service ORL et chirurgie cervico-faciale, Centre Hospitalier Emile Roux, F-43012, Le Puy en Velay, France

2-Université Jean Monnet, Faculté de Médecine, IFR INSERM 143 ; F-42023, Saint-Etienne, France.

3-LINA, EA 4624, F-42023, Saint-Etienne, France

4-Université de Lyon, F-42023, Saint-Etienne, France

5-DTF-Aerodrug, Faculté de médecine, F-37032, Tours, France

6-INSERM U-618 Protéases et Vectorisation Pulmonaires, Université François Rabelais, Faculté de médecine, F-37032 Tours, France.

7-Ecole Nationale Supérieure des Mines de Saint-Etienne, Centre Ingénierie et Santé, IFR Inserm 143, F-42023 Saint-Etienne, France.

8-Service de Médecine Nucléaire, CHU Saint-Etienne, F-42055 Saint-Etienne, France.

9-Laboratoire de bactériologie, CHU de Saint-Etienne, F-42055 Saint-Etienne, France.

10-DTF-médical, 19 rue de la presse, BP 132, F-42003 Saint-Etienne, France.

11-Service d'Otorhinolaryngologie, Cliniques universitaires Saint-Luc, Université Catholique de Louvain, B-1200, Bruxelles, Belgique.

12-Service de Médecine Nucléaire, Cliniques universitaires Saint-Luc, Université Catholique de Louvain, B-1200, Bruxelles, Belgique.

13-Service de pneumologie, Cliniques universitaires Saint-Luc, Université Catholique de Louvain, B-1200, Bruxelles, Belgique.

* Auteur correspondant :

Adresse e-mail : marc.durand@ch-lepuy.fr (M. Durand) 
Résumé

Objectifs: L'administration nasale de médicaments sous forme d'aérosol est largement utilisée par les oto-rhino-laryngologistes. Bien qu'elle manque d'évaluations cliniques, la nébulisation ORL se présente comme une option thérapeutique intéressante pour l'administration nasale de médicaments. Les aérosols permettraient de cibler les sites anatomiques d'intérêt situés derrière la valve nasale. Le nébuliseur sonique NL11SN utilise l'addition d'un son de $100 \mathrm{Hertz}(\mathrm{Hz})$ à l'aérosol produit afin d'améliorer le dépôt dans les sinus. L'objectif de notre étude était : (1) d'évaluer in vivo chez le volontaire sain l'influence du son sur la ventilation sinusienne et le dépôt nasal et pulmonaire d'un aérosol sonique, et (2) de quantifier in vitro le dépôt d'aérosols soniques dans les sinus maxillaires d'un modèle plastiné.

Matériel et méthodes: In vivo, une scintigraphie de ventilation au gaz ${ }^{81 \mathrm{~m}} \mathrm{Kr}$ et une scintigraphie de dépôt d'aérosol $\left({ }^{99 \mathrm{~m}} \mathrm{Tc}-\mathrm{DTPA}\right)$ sonique produit par le NL11SN étaient réalisées chez 7 volontaires sains. En parallèle, des nébulisations de gentamicine étaient réalisées en présence ou en absence du son de $100 \mathrm{~Hz}$, sur un modèle de tête humaine plastinée avec le NL11SN. La quantité de gentamicine déposée dans les sinus était dosée par FPIA.

Résultats : L'addition du son de $100 \mathrm{Hertz}$ au gaz ${ }^{81 \mathrm{~m}} \mathrm{Kr}$ permettait la ventilation des cavités sinusiennes des volontaires sains. Le ${ }^{99} \mathrm{~m}$ Tc-DTPA nébulisé avec le NL11SN chez les volontaires se déposait majoritairement dans les cavités nasales $(2 / 3$ vs. $1 / 3$ dans les poumons). In vitro, le NL11SN utilisé en mode sonique a permis d'augmenter d'un facteur 3 $(p<0,002)$ le dépôt de gentamicine dans les sinus du modèle plastiné. Les quantités d'antibiotiques déposées dans les sinus du modèle plastiné pouvaient être considérées comme suffisantes pour avoir un effet thérapeutique local. 
Conclusions : Le nébuliseur NL11SN assure un dépôt préférentiel de l'aérosol dans les cavités nasales et cible efficacement les sinus maxillaires.

Mots clés :

Aérosol sonique ; $100 \mathrm{Hertz} \mathrm{;} \mathrm{scintigraphie} \mathrm{;} \mathrm{volontaire} \mathrm{sain} \mathrm{;} \mathrm{tête} \mathrm{plastinée} \mathrm{;} \mathrm{nasal} \mathrm{;} \mathrm{sinus} \mathrm{;}$ rhinosinusite 
Aim: Intranasal aerosol administration of drugs is widely used by ENT specialists. Although the clinical evidence is still missing, intranasal nebulization appears as a promising therapeutic option for local delivery of drugs. Aerosols would enable to target the anatomic sites located beyond the nasal valve. The sonic nebulizer NL11SN uses an additional 100 Hertz $(\mathrm{Hz})$ sound to the aerosolization in order to improve the deposition in the nasal/paranasal sinuses. The aim of our study was 1) to evaluate in normal volunteers the influence of adding the $100 \mathrm{~Hz}$ sound on the sinusal ventilation and on the nasal and lung deposition of that kind of aerosol, and 2) to experimentally quantify the aerosol deposition in the maxillary sinuses in a model of plastinated head.

Material and methods: In vivo, scintigraphy of ventilation with ${ }^{81 \mathrm{~m}} \mathrm{Kr}$ gas and of sonic aerosol deposition $\left({ }^{99 m}\right.$ Tc-DTPA) using NL11SN, were performed in 7 healthy volunteers._In parallel, gentamicin nebulizations were performed with NL11SN, with or without adding the $100 \mathrm{~Hz}$ sound, on a model of plastinated human head; the gross amount of gentamicin delivered to the paranasal sinuses was determined using an FPIA method.

Results: The addition of the $100 \mathrm{~Hz}$ sound to the nebulization allowed ventilation of the paranasal sinuses in healthy volunteers studied with ${ }^{81 \mathrm{~m}} \mathrm{Kr}$ gas. The deposition of ${ }^{99 \mathrm{~m}} \mathrm{Tc}-\mathrm{DTPA}$ particles nebulized with NL11SN was noted predominantly in the nasal cavities $(2 / 3 v s .1 / 3$ in the lungs). In vitro, the use of NL11SN in sonic mode proved to increase threefold the gentamicin deposition in the sinuses of the plastinated model $(P<0.002)$. The amount of antibiotic deposited in the plastinated model may be considered sufficient to induce a local therapeutic effect.

Conclusion: The NL11SN nebulizer is able to ensure a preferential deposition of an aerosol in the nasal cavities and to target the maxillary sinuses. 


\section{Keywords:}

sonic aerosol ; $100 \mathrm{Hertz} \mathrm{;} \mathrm{scintigraphy} \mathrm{;} \mathrm{healthy} \mathrm{volunteers} \mathrm{;} \mathrm{plastinated} \mathrm{head} \mathrm{model} \mathrm{;} \mathrm{nasal} \mathrm{;}$ sinus ; rhinosinusitis. 


\section{Introduction}

Bien que l'efficacité de la nébulisation d'antibiotiques et de corticoïdes à visée pulmonaire soit démontrée par de nombreuses études [1,2], la nébulisation d'antibiotiques à visée nasale reste controversée [3,4]. En effet, l'AFSSAPS n'a pas retenu d'indication aux antibiotiques dans les pathologies rhinosinusiennes par voie locale [5]. Néanmoins, l'avantage théorique de la nébulisation par rapport aux voies classiques d'administration est de délivrer le médicament directement à l'organe cible afin de s'affranchir des effets secondaires systémiques, tout en augmentant localement la dose administrée. Par conséquent, même si peu d'évaluations cliniques ont été conduites et que le nombre d'études publiées à ce sujet est limité, la nébulisation à visée nasale est un mode d'administration de médicaments fréquemment utilisé par les ORL et par les généralistes [6,7]. Administrée dans la plupart des états inflammatoires aigus ou chroniques des cavités nasales ou sinusiennes, l'aérosolthérapie par voie nasale permet l'application du produit actif directement au niveau de la muqueuse rhinosinusienne, ainsi qu'une réponse clinique rapide en minimisant les effets secondaires. Elle serait plus particulièrement utilisée dans des cas où les traitements de référence par spray ou per os sont mis en échec [6,8]. La nébulisation pourrait se présenter en effet comme une option thérapeutique intéressante pour l'administration d'antibiotiques par voie nasale $[9,10]$.

Aucun spray nasal ne dispose actuellement de formulation antibiotique. De plus ces dispositifs semblent inadaptés au ciblage des sites anatomiques potentiellement infectés comme les sinus maxillaires, les cellules ethmoïdales, les cornets moyens [11,12]. Or l'utilisation de nébuliseurs, produisant des particules de taille plus fines que les sprays $(4 \mu \mathrm{m}$ vs. $30 \mu \mathrm{m})$, permet de cibler ces zones anatomiques situées après la valve nasale, mais ils restent néanmoins inefficaces pour atteindre les sinus maxillaires. Par contre, l'utilisation d'une onde acoustique de $100 \mathrm{Hertz}(100 \mathrm{~Hz})$ couplée à des aérosols nébulisés permettrait d'améliorer la ventilation [13] et le dépôt des aérosols [14] dans les cavités naso-sinusiennes. 
Les nébuliseurs soniques à visée nasale apparaissent en conséquence comme étant les générateurs d'aérosols les plus adaptés pour le traitement local des rhinosinusites.

L'objectif de cette étude est de quantifier l'aérosol déposé dans les voies respiratoires et dans les sinus maxillaires, produit par un nébuliseur sonique à visée nasale. Nous avons utilisé le nébuliseur NL11SN (DTF, Saint Etienne) spécialement conçu pour optimiser le dépôt de médicaments dans les cavités nasales et dans les sinus maxillaires, en additionnant à l'aérosol produit un son à $100 \mathrm{~Hz}$ [15]. Ce travail présente les résultats de l'évaluation de ce système de nébulisation sonique par voie nasale (i) in vivo, chez le volontaire sain avec un traceur radioactif et (ii) in vitro dans un modèle de tête humaine plastinée avec de la gentamicine.

\section{Matériel et Méthodes}

\subsection{Nébuliseur sonique nasal}

La nébulisation était réalisée avec un nébuliseur Sonique Nasal ATOMISOR NL11SN (NL11SN), associé au compresseur ATOMISOR AOHBOX® (DTF médical, Saint-Etienne, France). L'aérosol était administré via des embouts narinaires souples C28E (DTF médical, Saint-Etienne, France).

2.2. Etude scintigraphique chez les volontaires sains.

\subsubsection{Population de l'étude}


Etaient inclus dans cette étude monocentrique en tant que volontaires sains, sept hommes nonfumeurs âgés de 21 à 36 ans, d'une taille moyenne de $181 \pm 3 \mathrm{~cm}$ et d'un poids moyen de 77 $\pm 10 \mathrm{~kg}$.

Le protocole de l'étude a été approuvé par le comité d'éthique des Cliniques Universitaires Saint Luc de l'Université Catholique de Louvain (UCL, Belgique), lieu de déroulement de l'étude. En application de la déclaration d'Helsinki et en accord avec les bonnes pratiques cliniques, les volontaires sains ont donné et signé un consentement éclairé avant d'être définitivement inclus dans l'étude.

Les participants ont été inclus en tant que sujets sains lors de la première visite de sélection après un examen médical complet : examen physique, mesure des signes vitaux, recueil des antécédents médicaux et chirurgicaux, réponses aux critères d'inclusion et d'exclusion. En effet, du fait de la réalisation de scintigraphies pulmonaires, l'étude incluait uniquement des hommes d'âge, de taille et de poids équivalents afin de limiter et d'homogénéiser, au sein de la population, l'atténuation tissulaire du thorax.

Les principales causes d'exclusion dans l'étude étaient les suivantes : antécédents cardiaques et vasculaires, antécédents d'allergie, asthme ou autre pathologie pulmonaire, ainsi que tout antécédent de pathologies ORL, en particulier rhinosinusienne (polypose, tout types de rhinosinusites) et de chirurgie ORL ou cervico-faciale (réparatrice ou fonctionnelle).

L'examen clinique des volontaires sains était complété par une rhinoscopie des fosses nasales.

Le sujet 3 présentait une déviation nasale droite et le sujet 5, un éperon septal droit. Considérées comme des variantes anatomiques, ces observations cliniques ne justifiaient pas l'exclusion de ces sujets.

2.2.2. Etude de l'influence du son à 100 Hertz par scintigraphie de ventilation 
Une ventilation au gaz Krypton $81 \mathrm{~m}\left({ }^{81 \mathrm{~m}} \mathrm{Kr}\right)$ était réalisée chez les volontaires afin d'étudier l'influence de l'addition du son à 100 Hertz sur la pénétration du gaz dans les sinus maxillaires ainsi que pour déterminer les régions anatomiques des voies aériennes supérieures et des poumons. Le gaz ${ }^{81 \mathrm{~m}} \mathrm{Kr}$ (générateur de ${ }^{81} \mathrm{Rb} /{ }^{81 \mathrm{~m}} \mathrm{Kr}$ Covidien, Petten, Pays Bas) était administré en continu chez les volontaires, via l'embout narinaire, permettant ainsi la ventilation des voies aériennes supérieures et inférieures.

Les acquisitions scintigraphiques (2min) suivantes étaient réalisées à l'aide d'une gamma caméra STARPORT 400 AC/T (General Electric, Horsholm, Danemark) :

- Gaz-1 : les cavités nasales selon une vue latérale droite de la tête,

- Gaz-2 : les cavités nasales selon une vue de face de la tête, ventilation sans son,

- Gaz-3 : les cavités nasales selon une vue de face de la tête, ventilation avec son à 100 $\mathrm{Hz}$

- $G a z-4$ : les poumons selon une vue postérieure du thorax.

2.2.3. Etude du dépôt d'un aérosol sonique de ${ }^{99 \mathrm{~m}}$ Tc-DTPA

Le nébuliseur NL11SN était chargé avec $3 \mathrm{ml}$ d'une solution contenant 25mg de DTPA (Diethylène Triamine Penta Acetic Acid) marqué avec $74 \mathrm{MBq}$ de Technetium $99 \mathrm{~m}\left({ }^{99 \mathrm{~m}} \mathrm{Tc}\right)$ (TechneScan DTPA, Mallinckrodt Medical, Petten, Pays Bas).

Avant la séance d'aérosol, les volontaires étaient entraînés à inhaler l'aérosol par le nez puis à expirer par la bouche. Un système de filtres absolus (BB50TE, Pall medical, France) était utilisé pour assurer la protection de l'air ambiant et pour quantifier l'activité de l'aérosol exhalé. La nébulisation était réalisée avec addition de son à $100 \mathrm{~Hz}$ durant 10 minutes. Immédiatement après l'inhalation, le sujet s'asseyait face à la caméra et trois images étaient enregistrées : 
- Aérosol-1 : les cavités nasales selon une vue latérale droite de la tête,

- Aérosol-2 : le thorax selon une vue postérieure,

- Aérosol-3 : l'activité résiduelle du nébuliseur et des accessoires.

\subsubsection{Traitement des images}

L'activité totale déposée dans les voies respiratoires des volontaires était calculée par la méthode «activity balance » consistant à soustraire l'activité non déposée de l'activité initiale introduite dans le nébuliseur pour déterminer l'activité totale déposée dans les voies respiratoires. Les calculs étaient corrigés pour le bruit de fond et la décroissance radioactive. Des régions était tracées autour des zones anatomiques d'intérêt (cavité nasales, poumons, estomac) à partir des images de ventilation puis appliquées sur chacune des images de dépôt d'aérosol. Le pourcentage de dépôt d'aérosol dans chacune des régions d'intérêt était calculé à partir de l'activité totale déposée. La masse de DTPA déposée (en $\mu$ g) était également déterminée pour chacune des régions, à partir de la masse initiale de DTPA chargée dans le nébuliseur.

\subsection{Etude in vitro du dépôt d'un aérosol sonique de gentamicine}

Cette étude visait à comparer les concentrations intra sinusiennes d'un antibiotique, la gentamicine, nébulisé en présence ou en absence de son de $100 \mathrm{~Hz}$, dans un modèle anatomique de tête plastinée.

\subsubsection{Modèle anatomique de tête plastinée}

La technique de plastination de têtes humaines développée et adaptée à la modélisation ORL dans le laboratoire d'anatomie de la faculté de Médecine de Saint-Etienne depuis plus de dix ans a permis l'élaboration de plusieurs spécimens plastinés dont trois ont été dédiés à la réalisation d'études fonctionnelles des cavités naso-sinusiennes [16,17]. 
L'obtention des pièces anatomiques plastinées était réalisée selon les étapes habituelles de cette technique : préparation de la pièce (3 jours), fixation au formol (3 mois), déshydratation et dégraissage à l'acétone (1-2 mois), imprégnation forcée de silicone (10-20 jours), polymérisation (2-3 mois). L'étape de polymérisation a été optimisée pour éviter les phénomènes de rétraction tissulaire [17]. Les têtes plastinées ont été validés en tant que modèles d'études de dépôt d'aérosol après caractérisation anatomique, géométrique et aérodynamique, par les techniques classiques utilisée en clinique : rhinoscopie, endoscopie, tomodensitométrie, rhinomanométrie et rhinométrie acoustique.

Les sinus maxillaires des modèles ont été latéralement ouverts selon une coupe sagittale emportant la paroi latérale du sinus. Deux plaques de plexiglas amovibles étaient utilisées pour refermer hermétiquement les sinus lors des séances de nébulisation (Figure 1). Cet aménagement permettait d'accéder à l'antre du sinus maxillaire et de permettre la collection de l'aérosol déposé.

\subsubsection{Nébulisations de gentamicine}

La gentamicine (gentallin ${ }^{\circledR}$, Schering-Plough SAS, Courbevoie, France), aminoglycoside actif sur les bactéries à Gram négatif et sur les staphylocoques, dont l'efficacité sur les germes impliqués dans les rhinosinusites est reconnue [18,19] était choisie comme marqueur, en raison de sa solidité et de sa spécificité à pouvoir être dosée par des méthodes de routine. Deux concentrations étaient utilisées : $40 \mathrm{mg} / \mathrm{ml}$ et $80 \mathrm{mg} / \mathrm{ml}$.

Le nébuliseur NL11SN était chargé avec $4 \mathrm{ml}$ de gentamicine puis connecté sur les narines de la tête plastinée via l'embout narinaire (Figure 1).

La séance de nébulisation était réalisée durant 10 minutes. Les plaques de plexiglas étaient ensuite retirées puis les sinus maxillaires étaient rincés à 4 reprises avec $1 \mathrm{ml}$ de sérum 
physiologique (avec une seringue) afin de recueillir la gentamicine déposée sur les parois des sinus. Le liquide de rinçage était conservé à $-20^{\circ} \mathrm{C}$ pour analyse ultérieure de la quantité de gentamicine recueillie.

Après chaque nébulisation, le spécimen de tête plastinée était lavé à grande eau puis séché à l'air libre pendant 48 heures minimum.

Les nébulisations $(\mathrm{n}=112)$ étaient réalisées en mode sonique (avec son à $100 \mathrm{~Hz}$ ) ou en mode classique (sans son), pour chaque concentration en gentamicine. Des nébulisations de sérum physiologique $(n=34)$ étaient réalisées afin de vérifier qu'il ne reste pas de résidus de gentamicine dans les sinus, et de s'assurer ainsi de la qualité du lavage du modèle.

\subsubsection{Dosage de gentamicine}

Au total 291 liquides de rinçage sinusien ont été analysés pour le dosage de la gentamicine, par une méthode immuno-enzymatique (FPIA) sur un automate TDX/FLX® (Abbott Diagnostics, Rungis, France). Les dosages de gentamicine étaient réalisés en triplicat avec les réactifs Gentamicin ${ }^{\circledR}$ (Abbott Laboratories, Diagnostic Division, EU) dont le seuil de sensibilité était de $0,27 \mathrm{mg} / \mathrm{L}$.

\subsection{Analyses statistiques}

L'effet du son à $100 \mathrm{~Hz}$ sur le dépôt sinusien de gentamicine dans le modèle de tête plastinée étaient analysés par des tests t (XLSTATS®). Le seuil de significativité était fixé pour un $\mathrm{p}<0,05$.

\section{Résultats}


In vivo, les séances d'inhalations (ventilation au gaz ${ }^{81 \mathrm{~m}} \mathrm{Kr}$ et nébulisations de ${ }^{99 \mathrm{~m}} \mathrm{Tc}-\mathrm{DTPA}$ ) étaient bien tolérées par chacun des volontaires sains et aucun événement indésirable n'a été rapporté. Les voies aériennes supérieures et inférieures étaient imagées à l'aide du gaz ${ }^{81 \mathrm{~m}} \mathrm{~K}$ pour chaque volontaire. Les images enregistrées montrent l'effet positif du son à $100 \mathrm{~Hz}$ couplé à la ventilation sur la pénétration du gaz dans les sinus maxillaires (Figure 2, Gaz-2 et Gaz-3).

Ces images permettaient également de définir les régions anatomiques des voies aériennes supérieures et des poumons, afin de quantifier le dépôt de l'aérosol sonique de ${ }^{99 \mathrm{~m}} \mathrm{TcDTPA}$ inhalé par voie nasale (Figure 1, Aérosol-1).

En termes de masse de ${ }^{99 \mathrm{~m}}$ Tc-DTPA, l'aérosol déposé dans les voies respiratoires des sujets se distribuait à $2400 \pm 475 \mu \mathrm{g}$ dans les voies aériennes supérieures (cavités nasales et rhinopharynx) et à $925 \pm 425 \mu \mathrm{g}$ dans les poumons, démontrant un ciblage préférentiel de l'aérosol dans les cavités nasales (2/3 de l'activité) par rapport aux poumons. Les calculs réalisés pour les voies aériennes supérieures tenaient compte de la déglutition et de la clairance nasale (Tableau 1).

Cependant, les images ne montraient qu'une faible quantité d'aérosol pénétrant dans les sinus maxillaires, peu équivalente à la pénétration du gaz krypton mise en évidence dans ces cavités au cours de la scintigraphie de ventilation. Dans ce cas, un traitement d'image valide visant à quantifier de façon précise le dépôt d'aérosol dans ces régions maxillaires ne pouvait être appliqué sur ces images, en raison de la difficulté à situer anatomiquement ces régions.

Peu de DTPA radioactif était quantifié dans l'estomac $(0 \pm 25 \mu \mathrm{g})$ indiquant que peu d'aérosol était dégluti par les volontaires pendant la séance de nébulisation.

In vitro, les 112 nébulisations de gentamicine et les rinçages répétés n'avaient pas altéré l'anatomie du modèle de tête plastinée. L'absence de gentamicine dans les sinus après rinçage 
était contrôlée par un dosage de gentamicine dans le liquide sinusien collecté après nébulisation au sérum physiologique (résultat $<0,27 \mathrm{mg} / \mathrm{l}$ ).

Les résultats détaillés dans le tableau 2 démontraient une augmentation jusqu'à un facteur 3 $(\mathrm{p}<0,05)$ de la concentration en gentamicine collectée dans les sinus maxillaires lorsque la nébulisation était réalisée avec addition du son à $100 \mathrm{~Hz}$ en comparaison de la nébulisation sans son, et ceci pour les deux types de concentrations testées. Le dépôt de gentamicine dans le sinus gauche était significativement supérieur au dépôt de gentamicine dans le sinus droit avec ou sans addition de son $(\mathrm{p}<0,05)$.

\section{Discussion}

L'intérêt d'additionner un son à $100 \mathrm{~Hz}$ à l'administration nasale a été dans un premier temps, étudié in vivo directement chez le volontaire sain, par scintigraphie de ventilation au gaz ${ }^{81 \mathrm{~m}} \mathrm{Kr}$. Le gaz était administré par voie nasale avec le nébuliseur NL11SN et la ventilation des cavités nasales était enregistrée, de face, avant puis pendant l'addition du son. Les images (Figure 2, Gaz-2 et Gaz-3) démontrent l'influence du son à $100 \mathrm{~Hz}$ sur la pénétration du gaz dans les sinus maxillaires des volontaires sains. La ventilation est favorisée en présence du son mettant en évidence une augmentation des échanges gazeux entre les cavités nasales et les sinus maxillaires.

Cependant, comparé à un gaz, un aérosol de médicament peut avoir un comportement aérodynamique différent et par conséquent être plus ou moins influencé par les vibrations acoustiques de $100 \mathrm{~Hz}$. Ainsi, nous avons étudié la distribution dans les voies respiratoires des volontaires de cet aérosol sonique inhalé par voie nasale en utilisant un traceur radioactif, le ${ }^{99 \mathrm{~m}}$ Tc-DTPA. La masse de ${ }^{99 \mathrm{~m}}$ Tc-DTPA déposée dans les voies aériennes supérieures et dans les poumons était calculée à partir de régions anatomiques définies sur les images de ventilation. L'aérosol sonique de ${ }^{99 m}$ Tc-DTPA se distribuait pour $2 / 3$ dans les voies aériennes 
supérieures et $1 / 3$ dans les poumons. Au sein des cavités nasales, l'aérosol se déposait du vestibule narinaire jusqu'au pharynx (Figure 2, Aérosol-1), et non pas uniquement dans les premiers $\mathrm{cm}$ des fosses nasales comme habituellement référencé dans la littérature [11] ce qui peut supposer un passage d'aérosol au niveau des méats moyens et inférieurs.

Néanmoins, cette étude in vivo présente certaines limites, l'une liée à la méthodologie et l'autre à l'inclusion de sujet sain. La méthodologie employée permet uniquement une quantification globale de l'activité déposée au niveau des voies aériennes supérieures sans pouvoir discriminer avec une grande précision le dépôt dans les régions thérapeutiques cibles comme les sinus maxillaires. L'étroite juxtaposition des sinus maxillaires avec les fosses nasales rend difficile la localisation anatomique exacte de ces régions au sein des voies aériennes supérieures [17,20], en raison notamment de l'Effet Compton. Dans ce contexte l'efficacité thérapeutique théorique de la quantité du médicament déposé dans les sites cibles ne peut être évaluée. Par ailleurs, le volontaire sain lui-même représente une limite à l'étude du ciblage de zones anatomiques d'intérêt thérapeutique. Celui-ci possède en effet des ostiums maxillaires ouverts (démontré par la ventilation au gaz ${ }^{81 \mathrm{~m}} \mathrm{Kr}$ ) ce qui n'est généralement pas le cas des patients présentant des pathologies rhinologiques. En effet, les patients qui pourraient être concernés par l'administration nasale d'antibiotiques dans le cadre d'une rhinosinusite chronique présentent pour la plupart un ostium fermé [21].

Dans un deuxième temps, nous avons étudié, in vitro, l'influence du son à $100 \mathrm{~Hz}$ additionné à un aérosol de gentamicine, dans un modèle de tête humaine plastinée. La plastination de tête humaine a permis la réalisation d'un concept de modèle offrant l'avantage premier de conserver la muqueuse, l'anatomie et l'aérodynamique des cavités nasales et des sinus. Le second avantage de ce modèle est de permettre la réalisation d'un dosage «in situ» des produits actifs nébulisés et déposés dans les sinus maxillaires. 
Les résultats obtenus montrent que la gentamicine nébulisée avec le NL11SN pénètre et se dépose dans les sinus maxillaires du modèle. Le principal facteur influençant le dépôt sinusien était le son. En effet, la concentration en gentamicine collectée dans les sinus était significativement augmentée d'un facteur 1,6 à 3 lorsque les nébulisations étaient réalisées en présence de son (Tableau 2). Pour une même charge de gentamicine, l'augmentation du dépôt sinusien avec le son est équivalente pour les deux sinus. Néanmoins, le dépôt est significativement asymétrique entre le sinus droit et le sinus gauche $(\mathrm{p}<0,05)$. Une géométrie différente des ostiums des sinus maxillaires pourrait être à l'origine de cette différence. En effet, les mesures effectuées sur ce même modèle par rhinomanométrie ont mis en évidence une résistance plus importante de l'ostium droit en comparaison du gauche [17,22]. Le gain sur le dépôt maxillaire de gentamicine apporté par le son était indépendant de la géométrie de l'ostium.

Bien que ce modèle présente l'avantage de pouvoir collecter les dépôts sinusiens, il présente néanmoins certaines limites. En effet, les résultats de dosage de la gentamicine présentent une grande variabilité. Celle-ci s'explique par la difficulté technique du rinçage du sinus au sérum physiologique pour collecter la gentamicine. En effet, bien que la technique soit maîtrisée, l'anatomie de l'antre du sinus et le dépôt non homogène de l'aérosol dans ces cavités ne permettent pas, d'une part de recueillir la totalité de la gentamicine déposée et, d'autre part d'obtenir un recueil de gentamicine strictement identique d'une expérience à l'autre.

De plus, le rinçage des sinus était suboptimal, puisqu'il n 'incluait ni la paroi latérale du sinus maxillaire, ni l'éventuelle adsorption physique de la gentamicine à la muqueuse sinusienne plastinée. Dans ce cas, la quantité de gentamicine réellement déposée dans les sinus est aussi certainement sous-estimée. 
Une limite technique liée à l'expérience doit être considérée, constituée par le fait que le spécimen de tête plastinée n'était pas ventilé, et par conséquent était «passif» face à l'administration de l'aérosol. En effet, l'inhalation et l'exhalation des particules aérosolisées n'étaient pas reproduites dans le modèle, pouvant entraîner une modification de la cinétique de dépôt de l'aérosol en comparaison avec un modèle ventilé. Cette absence de «respiration» peut être à l'origine d'une diminution de la pénétration de l'aérosol de gentamicine dans les sinus de la tête plastinée, ou, à l'inverse d'une augmentation. Une diminution d'une part, car en effet, les flux d'air générés au travers des cavités nasales durant les phases d'inspiration (accélération) et d'expiration (vortex) assurent un rôle dans le maintien de l'aération des sinus en permettant les échanges gazeux avec les cavités nasales (respiration sinusienne) [21,22,]. Une augmentation, d'autre part, due à une accumulation de l'aérosol dans les fosses nasales, favorisant sa pénétration dans les sinus maxillaires au travers des ostiums.

La nébulisation à visée nasale d'antibiotiques est controversée notamment à cause d'un manque de données sur les quantités de molécules actives réellement déposées, sur l'efficacité clinique potentielle de ce dépôt, mais aussi sur les doses thérapeutiques attendues.

Dans le cadre de notre étude, les résultats obtenus in vivo, chez le volontaire sain, et in vitro dans le modèle plastiné montrent que le nébuliseur sonique NL11SN est adapté au ciblage des voies aériennes supérieures dont les cavités sinusiennes.

Par ailleurs, le modèle de tête plastinée peut se montrer pertinent en première approche pour évaluer l'efficacité théorique du dépôt d'antibiotique obtenu dans les sinus maxillaires, en fonction des quantités de produits actifs recueillies. Ainsi dans notre étude, en moyenne 1,35 $\mathrm{mg} / \mathrm{L}$ et $2,33 \mathrm{mg} / \mathrm{L}$ étaient mesurés respectivement dans les liquides de rinçage des sinus droit et gauche, ce qui correspond à une masse moyenne de 3,31 $\mu \mathrm{g}$ recueillie dans les sinus. 
En termes d'efficacité de dépôt, il est possible d'estimer si cette quantité de gentamicine déposée peut être suffisante pour avoir un effet thérapeutique local. En effet, le dépôt sinusien de gentamicine peut être comparé avec le dépôt pulmonaire reconnu efficace d'un aminoglycoside de référence, en normalisant les dépôts par unité de surface tissulaire. Parmi les aminosides utilisés en nébulisation, la tobramycine (TOBI $\left.{ }^{\circledR}\right)$ est reconnue efficace dans le traitement des infections pulmonaires à Pseudomonas aeruginosa chez les patients atteints de mucoviscidose. Les études in vivo évaluent à environ $45 \mathrm{mg}$ le dépôt de tobramycine dans les poumons des patients obtenu avec le nébuliseur pneumatique de référence [1].

A partir de ces données, le rapport surfacique de dépôt de tobramycine dans les poumons peut être estimé à $0,0346 \mu \mathrm{g} / \mathrm{cm}^{2}$ (surface maximaliste de dépôt de $130 \mathrm{~m}^{2}$ ).

En assimilant le sinus maxillaire à une sphère d'environ $17 \mathrm{~cm}^{3}$ (en moyenne de 15 à $20 \mathrm{~cm}^{3}$ selon la littérature) [23,24,25], la surface potentielle pour le dépôt d'aérosol dans un sinus est égale à $31,93 \mathrm{~cm}^{2}$. Le dépôt de gentamicine obtenu dans les sinus de la tête plastinée est estimé à $0,05183 \mu \mathrm{g} / \mathrm{cm}^{2}$.

Selon ces nouvelles données, la quantité de gentamicine déposée dans les sinus du modèle in vitro est estimée comme étant 1,5 fois supérieure $\left(0,05183 \mu \mathrm{g} / \mathrm{cm}^{2}\right.$ vs. $\left.0,0346 \mu \mathrm{g} / \mathrm{cm}^{2}\right)$ en comparaison avec le dépôt pulmonaire de l'aminoside de référence. Ainsi, avec cette première approche, la quantité de gentamicine recueillie dans les sinus maxillaires semble être suffisante pour avoir un effet thérapeutique local au titre d'anti-infectieux. Ces résultats devront être confirmés par une étude clinique visant à évaluer l'efficacité thérapeutique du dépôt d'antibiotiques par aérosol sonique à visée nasale.

\section{Conclusions}

Ce travail montre que la nébulisation sonique, i.e. couplée avec un son à $100 \mathrm{~Hz}$, et comme réalisée avec le nébuliseur NL11SN, optimise le dépôt de l'aérosol dans les cavités nasales 
(2/3) et cible efficacement les régions anatomiques d'intérêt thérapeutique comme les sinus maxillaires. L'atteinte des sinus maxillaires prouve le passage par le méat moyen, centre de la pathologie sinusienne. Ainsi, les aérosols soniques à visée nasale produits par le NL11SN, pourraient être utilisés pour traiter certaines pathologies rhinosinusiennes.

En comparant avec des résultats de dépôt pulmonaire normalisés par unité de surface tissulaire, la quantité de médicament déposée dans les sinus peut être considérée comme suffisante pour avoir un effet thérapeutique anti-infectieux local. Le modèle de tête plastinée semble un modèle pertinent pour évaluer d'un point de vue thérapeutique l'efficacité théorique du dépôt sinusien de médicament, et en particulier d'antibiotiques. Ainsi les résultats montrent que l'utilisation d'antibiotiques en nébulisation rhinologique pourrait être utile, sans représenter de danger d'acquisition de résistance.

Des études cliniques sont indispensables pour valider l'efficacité thérapeutique du dépôt d'antibiotiques ou d'autres molécules par nébulisation, avec ce nébuliseur sonique (NL11SN), chez des patients présentant diverses pathologies rhinosinusiennes (infectieuses et/ou inflammatoires), et ainsi définir les applications cliniques. 
Conflit d'intérêt :

Sandrine Le Guellec: Chargée d'étude DTF-Aerodrug, employée de DTF.

Gilles Chantrel: Co-gérant de DTF.

Laurent Vecellio: Directeur scientifique DTF-Aerodrug, employé de DTF.

Le reste des auteurs n'ont déclaré aucun conflit d'intérêt. 
[1] Lenney W, Edenborough F, Kho P, Kovarik JM. Lung deposition of inhaled tobramycin with eFlow rapid/LC Plus jet nebuliser in healthy and cystic fibrosis subjects. Cyst Fibros 2011;10(1):9-14.

[2] Donnelly R, Seale JP. Clinical pharmacokinetics of inhaled budesonide. Clin Pharmacokinet 2001;40(6):427-40.

[3] Desrosiers MY, Salas-Prato M. Treatment of chronic rhinosinusitis refractory to other treatments with topical antibiotic therapy delivered by means of a large-particle nebulizer: results of a controlled trial. Otolaryngol Head Neck Surg. 2001;125(3):265-9.

[4] Videler WJ, van Drunen CM, Reitsma JB, Fokkens WJ. Nebulized bacitracin/colimycin: a treatment option in recalcitrant chronic rhinosinusitis with Staphylococcus aureus? A doubleblind, randomized, placebo-controlled, cross-over pilot study. Rhinology 2008;46(2):92-8.

[5] Antibiothérapie locale en ORL: Argumentaire. 3.3 Efficacité cliniques des antibiotiques locaux à usage nasal ou sinusal. Agence Française de Sécurité Sanitaire des Produits de Santé, Juillet 2004.

http://www.afssaps.fr/var/afssaps_site/storage/original/application/cf17911a89b9a62c4d777e 9a38a2abed.pdf.

[6] Serrano E. L'aérosolthérapie en ORL. Revue Française d'allergologie et d'immunologie clinique 2007;47:508-10.

[7] De Monte M, Scruignec J, Dubus JC, et al. N.U.A.G.E.S: a survey of nebulisation practice in France with regard to ERS guidelines. Respir Med 2007;101(12):2561-5.

[8] Fokkens WJ, Lund VJ, Mullol J. European Position Paper on Rhinosinusitis and Nasal Polyps. Rhinology 2007;45(suppl 20):1-139.

[9] Vaughan WC, Carvalho G. Use of nebulized antibiotics for acute infections in chronic sinusitis. Otolaryngol Head Neck Surg 2002;126:558-68.

[10] Scheinberg PA, Otsuji A. Nebulized antibiotics for the treatment of acute exacerbations of chronic rhinosinusitis. Ear Nose Throat J 2002;81(9):648-52.

[11] Suman JD, Laube BL, Dalby R. Comparison of nasal deposition and clearance of aerosol generated by nebulizer and an aqueous spray pump. Pharm Res 1999;16:1648-52.

[12] Laube B. Devices for Aerosol delivery to treat sinusitis. J.aerosol. med 2007;20 (1):5-18.

[13] Maniscalco M, Sofia M, Weitzberg E, Lundberg JO. Sounding airflow enhances aerosol delivery into the paranasal sinuses. Eur J Clin Invest 2006;36:509-13.

[14] Möller W, Schuschnig U, Meyer G, Mentzel H, Keller M. Ventilation and drug delivery to the paranasal sinuses: studies in a nasal cast using pulsating airflow. Rhinology 2008;46:213-20. 
[15] Guillerm R, Badre R, Flottes L, Riu R, Fey A. A new method for aerosol penetration into the sinuses. Presse Med 1959;30:1097-8.

[16] Durand M, Rusch P, Granjon D et al. Preliminary study of the deposition of aerosol in the maxillary sinuses using a plastinated model. J Aerosol Med 2001;14:83-93.

[17] Durand M, Pourchez J, Louis B et al. Plastinated nasal model: a new concept of anatomically realistic cast. Rhinology 2011;49(1):30-6.

[18] Kalogjera L, Vagić D, Baudoin T. Effect of endosinusal treatment on cellular markers in mild and moderate asthmatics. Acta Otolaryngol 2003;123(2):310-13.

[19] Bhattacharyya N, Kepnes LJ Assessment of trends in antimicrobial resistance in chronic rhinosinusitis. Ann Otol Rhinol Laryngol 2008;117(6):448-52.

[20] Aggarwal R, Cardozo A, Homer JJ. The assessment of topical nasal drug distribution. Clin Otolaryngol Allied Sci 2004;29:201-5.

[21] Eloy P, Nolevaux MC, Bertrand B. Physiologie des sinus paranasaux, EMC-Oto-rhinolaryngologie 2005;2:185-97.

[22] Croce $\mathrm{C}$, Fodil R, Durand $\mathrm{M}$ et al. In vitro experiments and numerical simulations of airflow in realistic nasal airway geometry. Ann Biomed Eng 2006;34:997-07.

[23] Emirzeoglu M, Sahin B, Bilgic S, Celebi M, Uzun A. Volumetric evaluation of the paranasal sinuses in normal subjects using computer tomography images: a stereological study. Auris Nasus Larynx 2007;34(2):191-5.

[24] Dah-Jouonzo H, Baron P, Faure J, Treil J, Borianne P. Volumetric measurments of maxillary basal bones and facial air cavities using 3D imagery. Internationnal Orthodontics 2005;3:269-289.

[25] Paganelli A, Michel J, Varoquaux A, Adalian P, Thomassin J, Dessi P. Etude des variations de volumes des sinus maxillaires en fonction de l'âge et du sexe des individus : applications fondamentales et cliniques potentielles. $117^{\mathrm{e}}$ congrès de la SFORL, Octobre 2010, Paris, page A106, référence P030. 
Figures

Plaques de plexiglas amovibles

Modèle de tête

plastinée

Nébuliseur NL11SN

avec embout narinaire

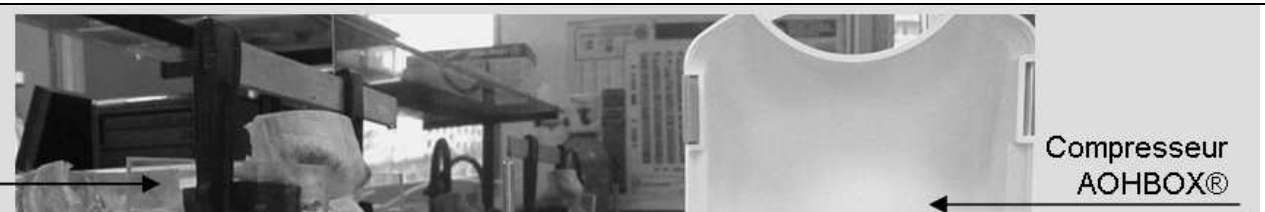

Flexible "son »

Flexible « pression »

Figure 1: Nébulisation de gentamicine dans un modèle de tête plastinée avec le nébuliseur sonique à visée nasale ATOMISOR NL11SN relié au compresseur ATOMISOR AOHBOX®. Le flexible «pression » assurait au nébuliseur une alimentation continue en air comprimé et le flexible «son» permettait la conduction du son de $100 \mathrm{~Hz}$ généré par le compresseur. Le nébuliseur sonique NL11SN était connecté aux narines de la tête plastinée par un embout narinaire. Les deux plaques de plexiglas amovibles disposées de chaque côté du modèle ferment hermétiquement l'accès extérieur aux sinus maxillaires. 

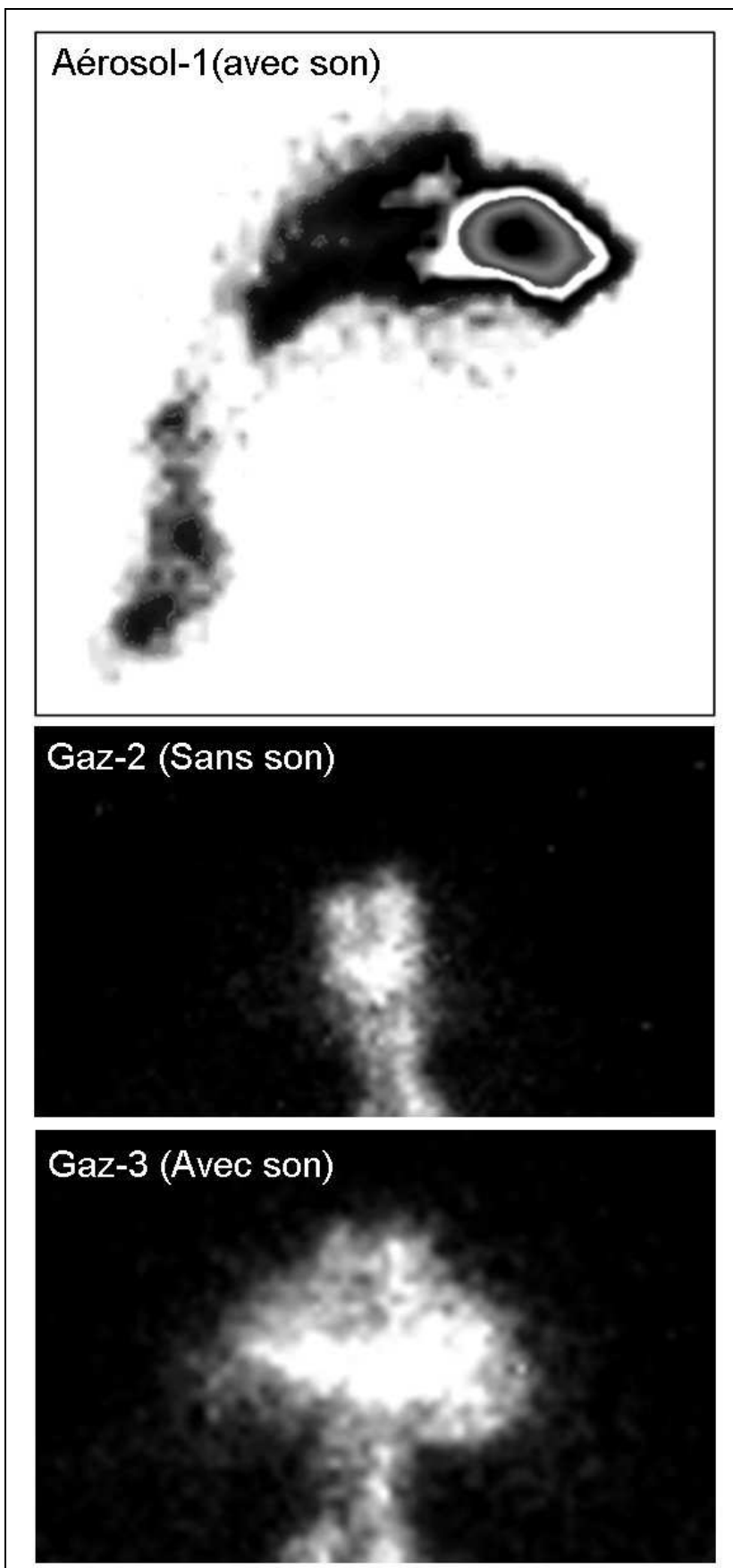

Figure 2: Images scintigraphiques obtenues chez les volontaires sains avec le nébuliseur sonique NL11SN/compresseur ATOMISOR AOHBOX® :

Aérosol-1 : dépôt d'aérosol de ${ }^{99 \mathrm{~m}}$ Tc-DTPA avec addition de son à 100 Hertz (vue latérale droite des cavités nasales),

Gaz-2 : ventilation au gaz ${ }^{81 \mathrm{~m}} \mathrm{Kr}$ sans addition de son (face antérieure des cavités nasales),

Gaz-3 : ventilation au gaz ${ }^{81 \mathrm{~m}} \mathrm{Kr}$ avec addition de son à 100 Hertz (face antérieure des cavités nasales). 
Tableau 1: Dépôt (en $\mu \mathrm{g}$ ) de l'aérosol de ${ }^{99 \mathrm{~m}} \mathrm{Tc}-\mathrm{DTPA}$ délivré par le nébuliseur NL11SN/compresseur AOHBOX®, obtenu chez les 7 sujets sains (moyenne \pm ET).

\begin{tabular}{l|l}
\hline Voies aériennes supérieures ${ }^{\text {a }}$ & $2400 \pm 475$ \\
Poumons & $925 \pm 425$ \\
Estomac & $0 \pm 25$ \\
\hline${ }^{\text {a }}$ Les voies aériennes supérieures tiennent compte des cavités nasales, du rhinopharynx \\
et de l'estomac.
\end{tabular}


Tableau 2: Concentrations moyennes $(\mathrm{mg} / \mathrm{l})$ en gentamicine collectée dans les sinus maxillaires droit et gauche du modèle de tête plastinée, obtenues après nébulisation classique (sans addition de son de $100 \mathrm{~Hz}$ ) ou sonique (avec addition de son de $100 \mathrm{~Hz}$ ) de deux formulations différentes de l'antibiotique $(40 \mathrm{mg} / \mathrm{ml}$ et $80 \mathrm{mg} / \mathrm{ml})$, par le nébuliseur NL11SN/compresseur AOHBOX®.

\begin{tabular}{|c|c|c|c|c|}
\hline \multirow{2}{*}{$\begin{array}{l}\text { Concentration en } \\
\text { gentamicine } \\
\text { chargée }(\mathrm{mg} / \mathrm{ml})\end{array}$} & \multirow[t]{2}{*}{$\begin{array}{c}\text { Type de } \\
\text { nébulisation }\end{array}$} & \multicolumn{2}{|c|}{$\begin{array}{l}\text { Concentration en gentamicine collectée } \\
\text { dans les sinus maxillaires }(\mathrm{mg} / \mathrm{l})\end{array}$} & \multirow{8}{*}{$\begin{array}{l}\mathrm{p}=0,679 * * \\
\mathrm{p}=0,242 * * \\
\mathrm{p}=0,005 * * \\
\mathrm{p}=0,019 * *\end{array}$} \\
\hline & & Sinus droit & Sinus gauche & \\
\hline 40 & classique & $<0,27^{\mathrm{a}}$ & $<0,27$ & \\
\hline 40 & sonique & $0,64 \pm 0,22$ & $0,73 \pm 0,32$ & \\
\hline \multicolumn{4}{|c|}{$\mathrm{p}=0,002 *$} & \\
\hline 80 & classique & $0,63 \pm 0,52$ & $1,19 \pm 1,03$ & \\
\hline 80 & sonique & $1,35 \pm 0,91$ & $2,33 \pm 1,49$ & \\
\hline \multicolumn{4}{|c|}{$\mathrm{p}<0,0001 *$} & \\
\hline
\end{tabular}

